\begin{tabular}{|c|c|c|c|c|c|}
\hline Cohort & Code & Gender & Age & Pituitary lesion & Surgical intervention \\
\hline \multirow[t]{5}{*}{ Control } & $\overline{C 1}$ & $\pi$ & 46 & - & - \\
\hline & $\mathrm{C} 2$ & $\hat{\sigma}$ & 47 & - & - \\
\hline & C3 & $\hat{0}$ & 32 & - & - \\
\hline & C4 & $\overline{0}$ & 28 & - & - \\
\hline & $\mathrm{C} 5$ & $\hat{0}$ & 38 & - & - \\
\hline \multirow[t]{5}{*}{ Acromegaly } & A1 & \& & 40 & Macroadenoma & Transsphenoidal \\
\hline & A2 & $\hat{0}$ & 42 & Macroadenoma & Transsphenoidal \\
\hline & A3 & 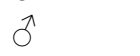 & 53 & Microadenoma & Transsphenoidal \\
\hline & A4 & $\hat{\sigma}$ & 55 & Macroadenoma & Transsphenoidal \\
\hline & A5 & $\hat{0}$ & 57 & Microadenoma & Transsphenoidal \\
\hline \multirow[t]{8}{*}{ Acromegaly + OCT } & O1 & 8 & 44 & Macroadenoma & Transsphenoidal \\
\hline & $\mathrm{O} 2$ & $\hat{0}$ & 55 & Macroadenoma & Transcranial \\
\hline & $\mathrm{O} 3$ & $\hat{O}$ & 56 & Macroadenoma & Transsphenoidal \\
\hline & $\mathrm{O} 4$ & $\hat{0}$ & 48 & Macroadenoma & Transsphenoidal \\
\hline & $\mathrm{O} 5$ & $\sigma^{\lambda}$ & 44 & Macroadenoma & Transsphenoidal \\
\hline & 06 & \& & 55 & Macroadenoma & Transsphenoidal \\
\hline & 07 & 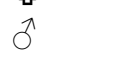 & 53 & Macroadenoma & Transsphenoidal \\
\hline & 08 & 8 & 68 & Macroadenoma & Transsphenoidal \\
\hline
\end{tabular}




\begin{tabular}{llll} 
Radiotherapy & \multicolumn{2}{l}{ Octreotide dose } & \multicolumn{2}{l}{ Current IGF-1 Current IC } \\
& $(\mathrm{mg})$ & $(\mathrm{ng} / \mathrm{dL})$ & ULN\% \\
\hline- & - & - & - \\
- & - & - & - \\
- & - & - & - \\
\hline- & - & - & - \\
- & - & - & - \\
\hline- & - & 223 & 0.76 \\
- & - & 180 & 0.81 \\
- & - & 180 & 0.81 \\
- & - & 183 & 0.81 \\
- & - & 166 & 0.73 \\
\hline- & 10 & 247 & 0.99 \\
- & 10 & 144 & 0.64 \\
- & 20 & 251 & 1.11 \\
- & 40 & 345 & 1.53 \\
+ & 30 & 284 & 1.26 \\
- & 30 & 232 & 1.22 \\
+ & 20 & 301 & 1.58 \\
- & 10 & 142 & 0.79 \\
\hline
\end{tabular}




\begin{tabular}{lll} 
Comorbidity & Colon polyp & Colorectal canı \\
\hline- & - & - \\
- & - & - \\
- & - & - \\
\hline- & - & - \\
- & - & - \\
\hline Diabetes insipitus & - & - \\
- & - & - \\
Hypogonadism, hypothyroidism, adrenal insufficiency & - & - \\
Hypogonadism & - & - \\
- & - & - \\
\hline- & - & - \\
Hypogonadism, hypothyroidism, adrenal insufficiency & + & - \\
- & + & - \\
- & + & - \\
- & - & - \\
- & - & - \\
Hypogonadism, hypothyroidism, adrenal insufficiency & + & - \\
Hypothyroidism & + & - \\
\hline
\end{tabular}




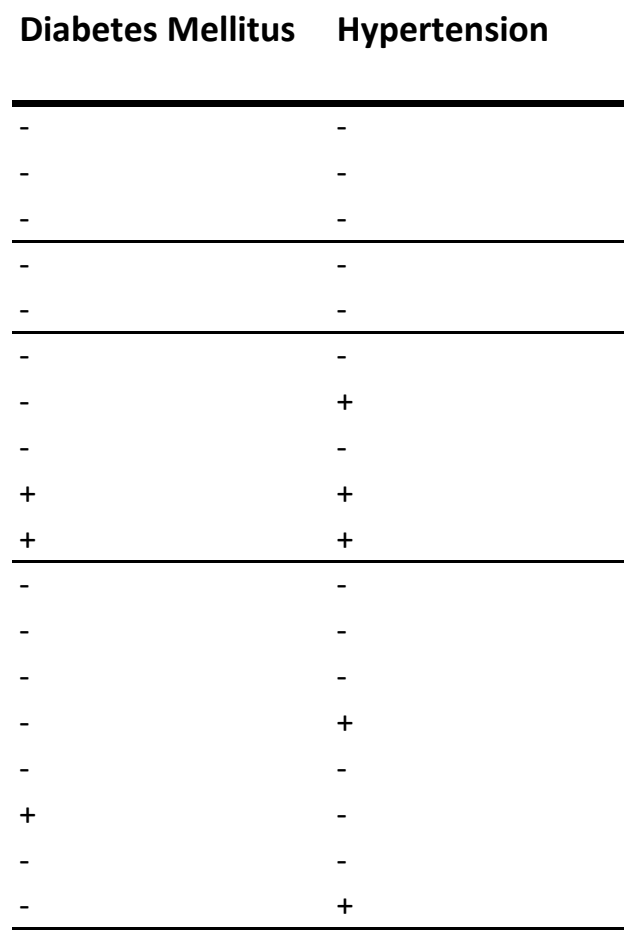




\section{Current medication}

\begin{tabular}{l}
\hline- \\
- \\
\hline- \\
\hline- \\
\hline Desmopressin po \\
Perindopril, amlodipine, doxazosin \\
Bisoprolol, prednisolone, testosterone, levothyroxine \\
Insulin, telmisartan, metformin, furosemid, thiazide, spironolacton \\
Metformin, pioglitazone, gliclazide, alpha lipoic acid, nebivolol \\
\hline- \\
Prednisolone, levothyroxine, cabergoline, calcium carbonate+vitamin D \\
Cabergoline, levothyroxine \\
Olmesartan, pegvisomant \\
Cabergolin, vitamin B12 \\
Metformin \\
- \\
Carvedilol, doxazosin, spironolacton, thiazide, citalopram, levothyroxine
\end{tabular}



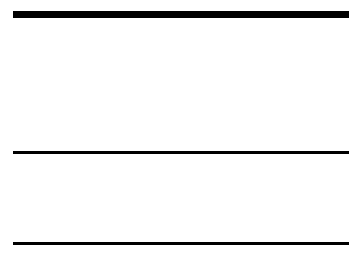

-

-

